\title{
Doctors' poor knowledge of nutritional supplementation in acutely unwell patients: no wonder recovery is slow!
}

\author{
M. I. Butt ${ }^{1}$, Katherine Barker ${ }^{2}$, Lynn Sawyer ${ }^{3}$ and Andrew Johnson ${ }^{4}$ \\ ${ }^{1}$ Diabetes Centre, Southmead Hospital, Monks Park Avenue, Bristol BS10 5NB, UK, ${ }^{2}$ Southmead Hospital, Monks Park \\ Avenue, Bristol BS10 5NB, UK, ${ }^{3}$ Southmead Hospital, Monks Park Avenue, Bristol BS10 5NB, UK and ${ }^{4}$ Southmead \\ Hospital, Monks Park Avenue, Bristol BS10 5NB, UK
}

\begin{abstract}
Aims: To assess the understanding of use of nutritional supplements by doctors.
Hypothesis: Doctors know when to appropriately prescribe nutritional supplements, when to discontinue their use and which supplements are clinically indicated.

Methods: We circulated the nutritional assessment questionnaire to all doctors irrespective of their grades working in our hospital through their hospital email addresses, distributing it at the grand round and journal club meetings.

Results: Sixty responses were received from a total of 132 giving a response rate of $46 \%$. These included foundation year one $(n=14$, $23.33 \%)$, foundation year two $(n=8,13.33 \%)$, senior house officers $(n=15,25 \%)$, specialist registrars $(n=14,23.33 \%)$ and consultants $(n=9,15 \%)$. Twenty percent $(n=12)$ of these responses were from geriatricians, $10 \%(n=6)$ from Endocrinologists, $6.67 \%(n=4)$ from Cardiologists, $5 \%(n=3)$ from Nephrologists and Respiratory physicians each and $53.33 \%(n=32)$ collectively from surgical, orthopaedics, haematology and ENT. Eighty-five percent $(n=51)$ of doctors regularly prescribed nutritional supplements to acutely admitted hospitalised patients. On a scale of ten, with ten being very important, all the doctors rated the nutritional status of hospitalised patients as important in their clinical management (average score 9.1/10). A majority of $90 \%(n=46)$ prescribed Fortisip supplements and onequarter of these doctors prescribed three supplements drinks per patient per day. Twenty-five percent of doctors $(n=15)$ prescribed Fortijuce with twice daily dosing as the most popular prescription $(47 \%)$. Eight percent $(n=5)$ prescribed Ensure, which is a non-stock product. Thirteen percent of doctors could correctly identify the calorie content of the Fortisip, and $2 \%$ doctors were able to identify its protein content. None knew the macronutrients of Calshake or Calogen. Only $7 \%(n=4)$ of the doctors prescribed nutritional supplements following advice from a dietician. Poor dietary intake $(85 \%, n=51)$, low albumin $(65 \%, n=39)$ and evidence of weight loss $(60 \%$, $n=36)$ were the most common reasons for prescription. Supplements were stopped following improved dietary intake $(78.3 \%, n=47)$, patient's refusal $(55 \%, n=33)$, albumin normalizing $(28 \%, n=17)$ and dietician advice $(3 \%, n=2)$. Seventy two percent of the participants $(n=43)$ felt the need for further education to understand the nutritional needs of acutely ill patients.

Summary and conclusions: We identified that majority of doctors $(93.77 \%)$ were prescribing the nutritional supplements without any prior discussions with the specialist dietician. Overwhelming majority did not know the caloric value and protein content of the prescribed supplements but were still prescribing them without the specialist dietician advice. Twenty percent of the doctors were prescribing Fortisip at a dose higher than the recommended daily dose. Majority of the participants felt further education about nutritional needs of patients.
\end{abstract}

